\title{
Literatura, ciencia y evolución: análisis de una experiencia educativa en Galápagos
}

\author{
Nicolás Cuvi ${ }^{1}$, Cristina Georgii ${ }^{2}$ \\ ${ }^{1}$ Programa de Estudios Socioambientales, Facultad Latinoamericana de Ciencias Sociales, FLACSO \\ Ecuador.ncuvi@flacso.edu.ec, ${ }^{2}$ Fundación CharlesDarwin.Ecuador, cristina.georgï@fcdarwin.org.ec
}

[Recibido en mayo de 2012, aceptado en enero de 2013]

\begin{abstract}
En este artículo se analiza una experiencia de divulgación y enseñanza de la teoría de la evolución realizada con estudiantes de ciencias naturales y literatura, en el archipiélago de Galápagos, mediante el uso del libro de ciencia en ficción titulado El misterioso reloj de Darwin (Cuvi, 2010). Se sostiene que la estrategia adoptada, la ciencia en ficción situada en contexto, es pertinente para la educación formal y no formal de adolescentes en temas como la teoría de la evolución, ecología, historia natural, geografía y conservación, necesarios para la sostenibilidad en un ámbito isleño y vulnerable como Galápagos. Se argumenta que el mestizaje del conocimiento, la construcción de una Tercera Cultura, la comprensión del mundo desde las simetrías, es materializada mediante la ciencia en ficción. Con esto no solo se construyen sociedades mejor conocedoras de su entorno y del funcionamiento de los sistemas de soporte de la vida, sino mejor alfabetizadas en los sentidos literario, científico y ecológico.
\end{abstract}

Palabras clave: ciencia en ficción; enseñanza de la evolución; medios alternativos de divulgación científica; Galápagos.

\section{Literature, science and evolution: analysis of an educational experience in Galapagos}

An experience of popularization and teaching of the theory of evolution among students of natural sciences and literature, in the Galapagos archipelago, through the the science-in-fiction book titled El misterioso reloj de Darwin (Darwin`s mysterious clock) (Cuvi, 2010), is analyzed. It is argued that the strategy adopted, science in fiction placed in context, is appropriate for the education (formal and informal) of adolescents on issues such as the theory of evolution, ecology, natural history, geography and conservation, necessaries for sustainability in an islander and vulnerable territory as Galapagos. It is argued that the cross-breeding of knowledge, the construction of a Third Culture, the understanding of the world from the symmetries, is materialized through science in fiction. This not only builds societies with a better knowledge of its environment and the function of the systems that support life, but more literate in literature, science and ecology.

Keywords: science in fiction; evolution teaching; alternative means of science popularization; Galapagos.

\section{Introducción: la ciencia en ficción como herramienta educativa}

Existe un consenso en torno a que la comprensión de la teoría de la evolución es pobre a escala mundial, y también de que, en varios lugares, los materiales existentes para enseñarla no cumplen con su objetivo (Tidon y Lewontin, 2004). Una de las dificultades que enfrenta la enseñanza de la evolución, en todos los contextos, se debe a las resistencias mediadas por las creencias religiosas y las perspectivas creacionistas, lo cual no sucede con otras ciencias exactas y naturales. Pero además, el aprendizaje de la teoría de la evolución es complejo porque, al igual que con la genética o la política ambiental, se carece de conocimiento previo suficiente (Alters y Nelson, 2002). Por estas dificultades, muchos esfuerzos pedagógicos, así como investigaciones, se orientan a debatir sobre nuevas estrategias y a entender los factores que son necesarios para educar sobre evolución; existe una vasta literatura en la cual se debaten dificultades, métodos, indicadores, estrategias, teorías pedagógicas, etcétera, que pueden ser aplicadas desde la escuela primaria hasta estudiantes universitarios de biología. Para estados de la cuestión véase: Alters y Nelson (2002) y Tidon y Lewontin (2004).

Si se considera que el conocimiento de la teoría de la evolución es crucial, entre otros aspectos, para promover la sostenibilidad ecológica (pues da cuenta de los procesos de largo plazo necesarios para garantizar la resiliencia de los sistemas de soporte de la vida), es 
pertinente ensayar nuevas formas de transmitirlo, entre las cuales la ciencia en ficción es una alternativa. El género de la ciencia en ficción ha sido popularizado por el químico y escritor Carl Djerassi, quien la explica de la siguiente manera:

"Denomino el género en que escribo "ciencia en ficción" para distinguirlo de la ciencia ficción. Como miembro que soy de una tribu, me exijo cierto grado de exactitud y verosimilitud que impriman a mi relato un elemento importante de realidad en la ficción" (Djerassi, 1996: 9).

"En oposición a la ciencia ficción, el género mucho menos frecuente de la ciencia en ficción tiene como base la ciencia verdadera o al menos razonable" (Djerassi, 2003: 9).

Con la ciencia en ficción, el científico sale de la posición en la que puede ser oscuro o indescifrable para el público, y se atreve a entablar una comunicación directa con éste, a quien considera un par (Erren y Falaturi, 2009). Ante este inusual público, el conocedor se ve obligado a encontrar recursos que trasciendan los registros tradicionales de la comunicación académica, por ejemplo la literatura, dándoles un valor simétrico. Un precursor clásico de esta estrategia fue Julio Verne, cuyas historias de aventura, hasta hoy, nos enseñan sobre aspectos científicos del mundo.

Erren y Falaturi (2009: 489) señalan además que "la ciencia bien investigada y embebida en diversas formas de ficción -ya sea seria, divertida o una mezcla de ambas- puede proporcionar información científica clave para el progreso cultural, información que proviene usualmente de lugares inesperados".

La ciencia en ficción, al considerar los diversos campos del conocimiento en simetría, es una vía para construir la Tercera Cultura, asunto debatido con intensidad por lo menos desde el referente trabajo de C.P. Snow (1959). Al construir esta Tercera Cultura, la ciencia en ficción mejora la comprensión pública de la ciencia y la tecnología, por lo cual también puede ser entendida como una estrategia para lograr una "ciencia para todos". Este diálogo entre las diferentes áreas del conocimiento humano ha sido propuesto y ejecutado en diferentes momentos de la historia por personajes como Voltaire, Da Vinci, Goethe, Heisenberg, Humboldt, entre otros. También en tiempos recientes, destacados biólogos como Stephen Jay Gould (2004) o Edward O. Wilson (1999) han apuntado a la necesidad de un diálogo entre ciencia y humanidades, aunque discrepando sobre la forma exacta en que debería ocurrir. En la actualidad proliferan iniciativas novedosas en este sentido, por ejemplo el Knowledge Incubation in Innovation and Creation for Science, liderado por la Red Europea de Centros y Museos de Ciencias (Ecsite por sus siglas en inglés) que "tiene como objetivo construir puentes entre las artes, la ciencia y la tecnología, proveyendo evidencia de los efectos positivos de esta interacción para la creatividad, así como para desencadenar interés en la ciencia" (Ecsite, 2012; traducción propia). O la iniciativa alemana Science in Dialogue, que considera a la ciencia en ficción como un eje fundamental para diseminar el conocimiento hacia audiencias amplias (Winter, 2004).

La ciencia en ficción es otra forma de comunicación de la ciencia y, más allá de los debates que puedan generarse en torno a los públicos de la ciencia y las estrategias para comunicarla (véase Nieto-Galan, 2011), no parece estar en discusión que la ciencia, en tanto cultura, debe circular.

La ciencia en ficción también puede ser entendida en el contexto de la relación ciencia-arte. Nos remitimos a un editorial de la revista Manguinhos, en el cual los editores, sin pretender agotar un tema tan amplio, dan cuenta del espectro de debates y casos que origina la confluencia entre arte y ciencia. 
"Las artes no tienen como función principal explicar o "ayudar" a las ciencias, ni éstas tienen como vocación elucidar a las primeras. El arte puede ser instrumental para la ciencia, pero no como muleta pedagógica: puede dejar claro su contenido bumano y contribuir para la construcción de su dimensión crítica. Por otro lado, la ciencia, cada vez más decisiva para la sobrevivencia de la bumanidad, si es utilizada adecuadamente, puede contribuir significativamente para la renovación de los elementos del quehacer artístico e inclusive como fuente inspiradora de sus creaciones" (Massarani, Moreira y Almeida, 2006: 10; traducción propia).

Este fructífero diálogo puede ser expandido al uso de la literatura, el teatro, el cine, u otras expresiones artísticas, para enseñar ciencias en espacios formales, articulación que consideramos positiva. Una obra de ciencia en ficción que aborde temas de historia natural, historia de la ciencia, teoría evolutiva, ecología, geografía y conservacionismo en Galápagos, nos parece, desde esta perspectiva, pertinente para la educación formal, al complementar (no reemplazar) los recursos existentes.

También la más convencional, comercial y difundida ciencia ficción es reconocida como herramienta para la educación científica en diversos casos, por ejemplo por Mateos (2004), Palacios (2007), y Sierra (2008).

La ciencia en ficción incluso ha sido usada como canal para plantear debates científicos de actualidad, por ejemplo por Osborne (1998). La hace poco tiempo lanzada Revista Ciencia, Público y Sociedad, editada en Argentina, incluye una sección de "Ficción Científica" o "Ciencia Ficción dura", preocupada por el avance de la tecnología y su rol en las sociedades. ¿Así también pueden ser expuestas y debatidas las teorías científicas? De acuerdo. Es más: así también deberían ser expuestas y debatidas las teorías científicas, para incluir al público no científico en el debate, y que éste pueda participar sin sentirse demasiado lejano de las evidencias empíricas y las propuestas teóricas. Por supuesto, esto no excluye la validez y la necesidad de los debates entre pares científicos, que ocurren en los canales tradicionalmente destinados a ello, como publicaciones y encuentros.

Conviene añadir que ciencia en ficción no equivale a divulgación de la ciencia en su acepción más pura. No es lo mismo una historia sobre las investigaciones en torno a la evolución, ateniéndose solamente a hechos reales, como el clásico y galardonado libro de Jonathan Weiner (1994) sobre los pinzones de Galápagos, que una historia que incluye elementos de ficción para conseguir similar objetivo.

Con estos antecedentes, la Fundación Charles Darwin (FCD) produjo el libro de ciencia en ficción titulado El misterioso reloj de Darwin (Cuvi, 2010), el cual fue entregado a estudiantes galapagueños de entre 10 y 15 años de edad, y a docentes de ciencias y de literatura, con el objetivo de promover, al mismo tiempo, una alfabetización científica, una alfabetización ecológica (Capra, 2002) y el fomento de una cultura literaria. Aspectos clave del libro fueron la transmisión del conocimiento sobre quién fue Charles Darwin y sobre diferentes aspectos de la compleja teoría de la evolución. Enseñar evolución no es algo fácil, ni en Galápagos ni en otros lugares del mundo (Tidon y Lewontin, 2004), pero en un tiempo de crisis de la biodiversidad, y en lugares especialmente vulnerables, es un reto que es necesario asumir desde enfoques plurales para ayudar a construir sociedades que sean más capaces de gestionar su entorno.

Luego de producir y distribuir el libro, nos propusimos analizar la experiencia, y el resultado de dicho proceso fue este artículo, cuyo objetivo central es compartir y debatir un proceso de enseñanza a través de un libro de ciencia en ficción. 


\section{El misterioso reloj de Darwin}

El libro El misterioso reloj de Darwin encaja bastante bien, aunque no perfectamente, en el género de ciencia en ficción. Además de tener rigor científico e histórico (características propias de la ciencia en ficción), incluye elementos de ciencia ficción, o no razonable, por ejemplo una máquina para viajar en el tiempo. Si bien esto pudo comprometer la exactitud científica de la obra en conjunto, fue una apuesta en aras de fomentar la comprensión de la evolución, de cumplir con un objetivo pedagógico.

En el libro se cuenta la historia de tres adolescentes galapagueños (los personajes centrales) que encuentran un objeto, el "misterioso reloj", que por un lado tiene la apariencia de un reloj antiguo, y por el otro tiene la pantalla y el teclado de un teléfono móvil. El objeto resulta ser una máquina del tiempo mediante la cual los adolescentes son transportados, dentro de una burbuja, a diferentes épocas y lugares, presenciando eventos que van desde la llegada de Charles Darwin a Galápagos en 1835, hasta los sofisticados estudios de pinzones realizados por Peter y Rosemary Grant en el islote Daphne Major, en nuestros días, pasando por los experimentos de Gregor Mendel, de Thomas H. Morgan, de Theodosius Dobzhansky, y de James Watson y Francis Crick. Para realizar cada viaje en el tiempo, los adolescentes deben resolver enigmas que aparecen en la pantalla. Al final de la historia se descubre que el misterioso reloj fue inventado por un científico con el objetivo de enseñar la teoría de la evolución a adolescentes, y que los protagonistas fueron sus sujetos experimentales.

Dejando de lado los puntuales detalles ficticios, lo que se narra en El misterioso reloj de Darwin sobre los científicos mencionados, y sobre la teoría evolutiva, es razonable. Por ello adherimos esta obra, en primera instancia, a la ciencia en ficción, aceptando la posibilidad de que el libro sea reclamado por, o enviado a, la literatura de divulgación científica; discusión que será fútil en última instancia, pues se trata de un híbrido entre literatura, divulgación y ensayo.

En relación con la teoría de la evolución, en el libro se comunican, entre otros, los cuatro puntos fundamentales para comprender este tema: variabilidad, herencia, selección y tiempo, de acuerdo con lo sugerido por Caldwell, Collins, Frankel, et al. (Sin fecha). Se abordan aspectos de herencia mendeliana, variación, síntesis evolutiva, biología molecular, ancestro común, radiación adaptativa, cladogénesis, selección natural, selección sexual, entre otros. Se enfatizan ciertos sucesos históricos que fueron fundamentales para que Darwin formulara su teoría, como su convencimiento de la pertinencia del gradualismo tras vivir un terremoto en Chile y tras sus observaciones de fósiles en los Andes. También se plantean debates sobre el creacionismo, la construcción de la ciencia, la valoración del científico y de su actividad; y se presentan aspectos de ecología como la contaminación o la conservación, y sobre la historia natural de algunas especies. El libro incluye, además, aspectos de geografía y geología del archipiélago.

En el libro, la historia, el arte, las ciencias de la vida, la geología, la geografía, la educación ambiental, la cultura científica, confluyen en simetría. El autor científico (Cuvi, 2010), biólogo e historiador de la ciencia, se comunica directamente con el público. Con El misterioso, más que una interacción o diálogo entre disciplinas (lo "interdisciplinar"), se consigue un mestizaje del conocimiento, cuestionando que el status quo de lo disciplinario en la educación formal sea la única vía para la transmisión de la cultura, manteniendo una mirada abierta, comprehensiva, compleja, holística, del pensamiento.

En el ámbito de la educación científica formal, El misterioso cumple con dos objetivos apuntados por Blanco (2004): "formación de científicos" y "mejora en el nivel de conocimientos de los ciudadanos". Asimismo, la obra se ubica en las tres esferas en las que, según el citado autor, ocurren relaciones entre educación y divulgación: el uso de productos de 
divulgación como producto didáctico en clase; el uso, por parte de los estudiantes, de productos de divulgación para su proceso de aprendizaje; y el estudio de los productos de divulgación como objetos de estudio en sí mismos.

El misterioso está escrito con una prosa propia de una novela de aventura. Tiene 14 ilustraciones realizadas por Alan J. Hesse, las cuales se aprovechan del humor para lograr su objetivo (por ejemplo la Ilustración 1, sobre la evolución de la iguana marina, endémica de Galápagos). Cuenta con 54 términos técnicos subrayados, los cuales constan definidos en un glosario.

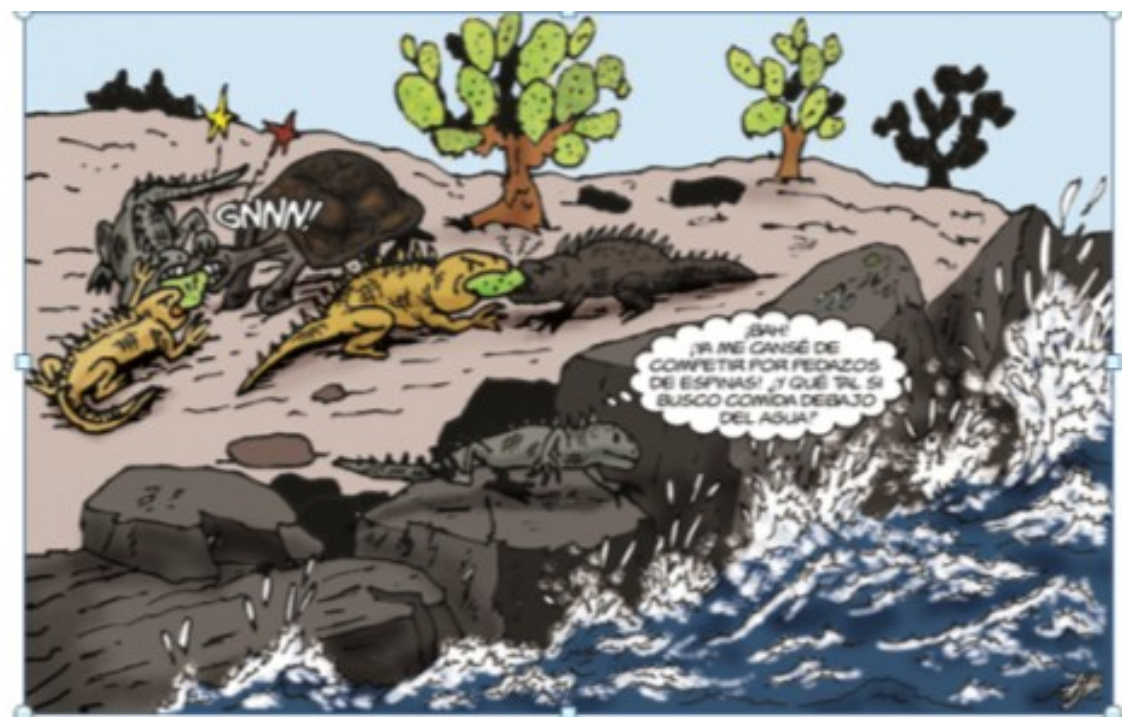

Ilustración 1. El cambio de estrategia en la selección de un nicho alimenticio permitió, entre otros aspectos, la evolución de la iguana marina. Ilustración: Alan J. Hesse, en Cuvi (2010).

\section{El archipiélago de Galápagos}

Ubicado en el Océano Pacífico, a mil kilómetros de la costa del Ecuador, el archipiélago está constituido por trece islas grandes, seis pequeñas, más de 40 islotes con nombres oficiales y decenas de pequeños roqueríos e islotes sin nombre (Jackson, 2007). El 97\% de su área terrestre es considerada Parque Nacional, y las aguas $20 \mathrm{~km}$ a la redonda tienen la categoría de Reserva Marina. Galápagos también es Patrimonio Natural de la Humanidad según la UNESCO.

Las principales actividades económicas son el turismo y la pesca; en el año 2010 vivían allí 25.124 habitantes (INEC, 2012) distribuidos en cuatro islas habitadas: Santa Cruz, San Cristóbal, Isabela y Floreana. Galápagos es la provincia con la mejor calidad de vida e ingreso del Ecuador, por lo que desde la década de 1980 ha tenido una fuerte inmigración, de entre el 4 y el 6\% anual, aproximadamente (Grenier, 2007). Esto ha ocasionado un intenso crecimiento urbano y de las amenazas a la biodiversidad y al ambiente en general. En 1998 se decretó una Ley Especial para regular, entre otros aspectos, la inmigración.

A comienzos del año lectivo 2011-2012 había 5.863 alumnos distribuidos en las cuatro islas. En San Cristóbal había 2.033 alumnos en diez planteles; en Isabela 593 alumnos en cinco planteles, en Santa Cruz 3.209 alumnos en nueve planteles (Ministerio de Educación, 2011c), y en Floreana una escuela unidocente hasta el $9^{\circ}$ grado de educación básica, con 28 estudiantes.

El archipiélago alberga una fauna endémica, sumamente mansa, que lo convierte en un sitio privilegiado para desarrollar estudios de evolución, ecología e historia natural. Esta fauna ilustra muy bien, por ejemplo, los procesos de radiación adaptativa a partir de un ancestro 
común. El contacto con las islas tuvo una decisiva influencia en Charles Darwin durante su elaboración de la teoría de la evolución por selección natural; el inglés señaló que una de las razones que le llevaron a indagar sobre la transmutación de las especies fue "el carácter sudamericano de la mayor parte de los productos del archipiélago de Galápagos, y muy especialmente la forma en que se diferencian ligeramente en cada isla del grupo" (Darwin, 1987: 109).

De forma paradójica, pese a que el nombre de Darwin es usado en Galápagos para nombrar calles, una isla, volcanes, centros educativos, restoranes, barcos, la estación científica y otros elementos culturales y geográficos, algunos moradores de las islas, muchos de reciente inmigración, desconocen quién fue dicho personaje. Algunos creen que es el jefe de la estación científica que lleva ese nombre, otros que fue uno de los primeros colonos, otros que fue un descubridor del archipiélago. En una encuesta realizada a 62 personas, el $71 \%$ respondió correctamente que fue un científico (Fundación Charles Darwin, 2008), y aunque la muestra no es significativa en relación con la población total del archipiélago, resulta un indicador interesante: tres de cada diez moradores no saben quién fue Darwin, menos aún su aporte a la ciencia.

La atención científica y el turismo que recibe Galápagos se deben a su naturaleza. Si se considera que el bienestar económico y social responde a la actividad turística, adquiere una mayor pertinencia promover la educación sobre la teoría de la evolución y sobre aspectos como la extinción o la vulnerabilidad de la biodiversidad. Al promover un mejor conocimiento de la naturaleza del archipiélago, se pueden fomentar actitudes más amigables hacia la misma, coadyuvando al objetivo de conservación. El conocimiento de la teoría de la evolución permite entender mejor otros aspectos de las ciencias de la vida, pues "nada tiene sentido en biología si no es a la luz de la evolución" (Dobzhansky, 1973). Una población conocedora de los procesos evolutivos de la naturaleza insular, será mucho más capaz de tomar decisiones en torno a su presente y su futuro.

También el Ministerio de Educación del Ecuador (2011a: 24) reconoce que la ecología y la evolución son dos aspectos fundamentales para "comprender las interrelaciones del mundo natural y sus cambios". Y aunque el contacto con la naturaleza no garantiza el aprendizaje de la evolución en particular, como demostró Bizzo (2008) en su estudio del entendimiento del tiempo geológico entre chicos que viven entre fósiles, sin duda es una ventaja que puede ser aprovechada.

\section{Metodología}

En junio de 2010 se entregaron 650 libros a estudiantes de las cuatro islas habitadas, durante ocho eventos de lanzamiento del libro con presencia del autor. Adicionalmente se distribuyeron 1.000 libros directamente a estudiantes y profesores de escuelas y colegios, y otros 350 a personal de la FCD, donantes, bibliotecas de instituciones educativas, sector público y ONG. Varios profesores de literatura y ciencias fomentaron su lectura como parte de sus clases.

Meses después nos preguntamos sobre la recepción de la obra, por lo cual entre septiembre y noviembre de 2010 (según la disponibilidad de cada institución educativa), se encuestó a 278 estudiantes que habían recibido el libro. La encuesta fue anónima y contaba con preguntas cerradas y abiertas.

Algunas preguntas que guiaron la investigación sobre la recepción de El misterioso reloj de Darwin entre el público estudiantil galapagueño fueron: ¿Qué conocimiento tienen los estudiantes sobre quién fue Charles Darwin y sobre aspectos relacionados con la teoría de la 
evolución? ¿Es la ciencia en ficción un recurso eficiente para apoyar y fortalecer a la educación formal en torno a la literatura, la historia y las ciencias de la vida en el contexto galapagueño? ¿Qué aprendizajes quedan de esta experiencia educativa? Y finalmente: ¿Cómo se articula esta experiencia con los debates en torno a la construcción de una Tercera Cultura?

Mediante la encuesta a estudiantes (Anexo 1) se indagó específicamente sobre: libros leídos previamente; alcance de la lectura de El misterioso reloj de Darwin; conocimiento de quién fue Charles Darwin y qué hizo en Galápagos; entendimiento de cómo llegaron las especies que habitan Galápagos; comprensión del concepto de la evolución y sobre cómo evolucionan los seres vivos; y finalmente opiniones de los lectores sobre la obra. Además, preguntamos a once profesoras sobre la utilidad del libro y su opinión de éste como herramienta educativa (Anexo 2).

Las encuestas, diseñadas por los autores del artículo, fueron validadas por una psicóloga en Quito y por una pedagoga en Galápagos, quienes hicieron sugerencias puntuales.

Si consideramos que se distribuyeron alrededor de 1.650 libros directamente a estudiantes, la muestra de encuestados correspondió al 17\% de quienes recibieron el libro. La muestra estuvo distribuida en tres islas (no en Floreana), ocho escuelas y cinco años escolares, como consta en el Gráfico 1. Esta muestra fue seleccionada de forma aleatoria, ateniéndonos a la disponibilidad de las profesoras y directivos de las escuelas, por lo que su distribución, en algunos aspectos, no permite comparaciones tan claras (por ejemplo, la segregación de datos por islas o por año lectivo). Esto de ninguna manera resta validez a la muestra, sino solamente alcance explicativo en aspectos puntuales. De todos modos, los análisis se hicieron sin extender los resultados a toda la población escolar; son indicativos únicamente de la muestra estudiada.

Las encuestas fueron tabuladas y clasificadas por la Máster en Biología Evolutiva, Paola Espinosa, utilizando una hoja de cálculo Excell. En el caso de las preguntas abiertas, se les asignó categorías ad hoc, como consta en los resultados más adelante. El análisis de la base de datos fue realizado con los filtros y conteos de la hoja de cálculo.

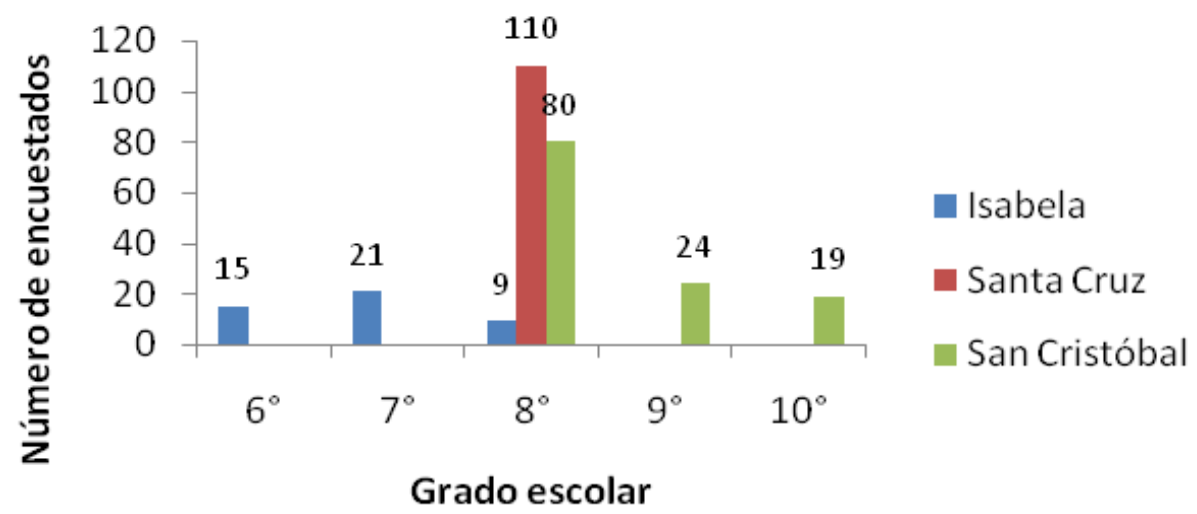

Gráfico 1. Distribución de la muestra de encuestados, según grado de escolaridad e isla.

\section{Resultados y discusión}

Preguntas 1 y 2: ¿Cuántos libros has leido? ¿Leiste El misterioso reloj de Darwin?

Era esencial saber si el libro había sido leído, y cuánto, para correlacionar esa información con otras variables, como hábitos previos de lectura, año escolar, escuela, isla de residencia, o conocimiento adquirido. 
El 29\% de estudiantes leyó todo el libro, el 24\% poco más de la mitad, el 32\% poco menos de la mitad, el 11\% sólo el comienzo y el $4 \%$ nada. A primera vista este porcentaje de lectura parece bajo, pero el resultado se convierte en un logro cuando se consideran los hábitos previos de lectura: el 33\% había leído más de cinco libros; el 16\% había leído entre 4 y 5 libros; el 34\% entre dos y tres libros; el 14\% uno solo; y el restante 2\% no había leído ningún libro. Si sólo la mitad había leído más de tres libros antes, un 50\% de lectores que llegaron a "más de la mitad" de El misterioso puede considerarse un porcentaje aceptable.

No apreciamos relación alguna entre el alcance de la lectura y otras variables como el año escolar, la isla de residencia, o el carácter privado o público de la institución educativa. Solamente detectamos una relación clara con la ideología religiosa: entre quienes no leyeron nada, o solamente el comienzo, se cuentan 15 alumnos del Colegio Adventista Loma Linda, del cual apenas dos estudiantes lo terminaron.

\section{Pregunta 3: ¿Quién fue Charles Darwin y qué hizo en su visita a Galápagos?}

Se planteó a los estudiantes una pregunta cerrada con cinco opciones, además de la posibilidad de dejar la respuesta en blanco. De la muestra total, el 82\% respondió correctamente (Gráfico 2), pero si se considera sólo a los de $9^{\circ}$ y $10^{\circ}$ grado, el $100 \%$ acertó. Esto bien podría estar asociado con el hecho de que ya han pasado por la educación obligatoria de estos temas.

El resultado, sea de la muestra total o de la sesgada por cursos, es positivo y prometedor, pues marca una diferencia entre estos adolescentes y el público que respondió a la encuesta de la Fundación Charles Darwin (2008). Aún así, el 18\% de respuestas equivocadas en la muestra total, refleja la necesidad de reforzar este conocimiento, aún antes de su estudio obligatorio, en un contexto donde conocer quién fue Charles Darwin y qué hizo en Galápagos resulta relevante, entre otros aspectos, para la construcción de una identidad asociada al territorio.

Para entender quién fue Darwin tras la lectura de El misterioso, bastaba leer "poco menos de la mitad del libro", por lo cual excluimos del análisis a quienes no leyeron nada, o solamente leyeron el comienzo, quedando 247 encuestados. De éstos, el 87\% dio una respuesta correcta. Este aumento del 5\% podría indicar un impacto positivo del contacto con el libro.
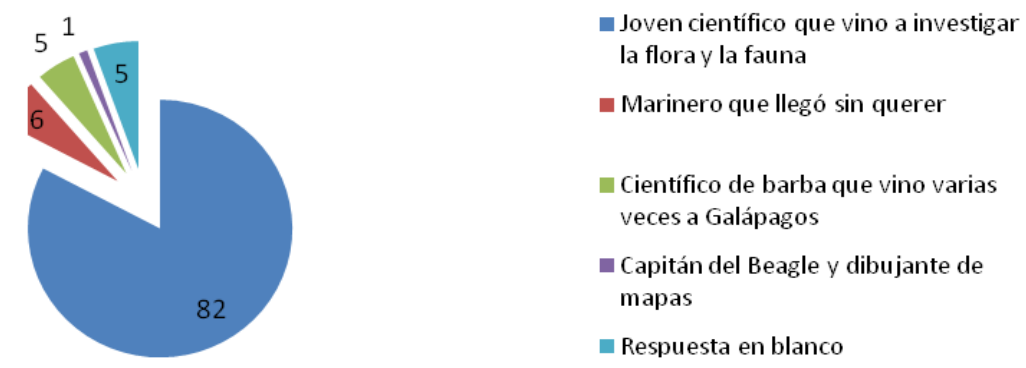

Gráfico 2. Respuestas ante la pregunta ¿Quién fue Charles Darwin y qué hizo en su visita a Galápagos? (porcentaje).

Nota: Nadie escogió la respuesta "Un turista".

\section{Pregunta 4: ¿Cómo llegaron los ancestros de las plantas y animales propios de Galápagos?}

Las estrategias de colonización de islas oceánicas como Galápagos son una evidencia indirecta de la teoría evolutiva, pues muestran que muchos ancestros de las especies llegaron desde masas continentales. Por eso se hizo la pregunta cerrada "¿Cómo llegaron los ancestros de las plantas y animales propios de Galápagos?", indicando que se debían escoger dos respuestas entre las siguientes: a) En barcos de piratas; b) En balsas de vegetación y arrastradas por los vientos; c) 
La gente los trajo; d) Siempre vivieron aquí; e) Volando, nadando, flotando. Las respuestas correctas eran $\mathrm{b}+\mathrm{e}$.

Entre las 271 respuestas de quienes leyeron por lo menos algo del libro, el 64\% contestó correctamente. Como esta información aparece en la mitad del libro, se consideró únicamente a quienes llegaron "por lo menos hasta la mitad del libro", aumentando el porcentaje de aciertos al $77 \%$. Al igual que en la pregunta anterior, el resultado sugiere que se dio una mejoría de conocimientos gracias al libro, aunque al mismo tiempo refleja un estado de conocimientos parcial.

Preguntas 5, 6 y 7: De acuerdo con la información del libro, ¿cómo evolucionan los animales y plantas?; Después de leer El misterioso reloj de Darwin, ¿qué es para ti la evolución?; Muestra con dibujos la teoría de la evolución de las especies.

Se plantearon dos preguntas abiertas que fueron agrupadas en un solo paquete de análisis: 1) De acuerdo con la información del libro, ¿cómo evolucionan los animales y plantas?, y 2) Después de leer el libro, ¿qué es para ti la evolución? Además, se pidió mostrar con dibujos lo que es la evolución.

En total, 214 estudiantes respondieron ambas preguntas, lo cual arroja el significativo dato de sesenta estudiantes que dejaron las respuestas en blanco. Las respuestas fueron agrupadas por la bióloga evolutiva en seis categorías de acierto y cuatro de desacierto. En algunos casos se asignó una respuesta a más de una categoría, dando como resultado 539 conceptos. La ubicación de una respuesta en categoría de acierto no obedeció a una "perfección" en su contenido; sólo se consideró la existencia de una noción adecuada, por general que fuera, atribuyendo el error solamente cuando se detectaba una verdadera confusión.

Las categorías de acierto representaron el 84\% de respuestas y fueron: adaptación a recursos y al clima; variación; cambios a través del tiempo; selección natural; concepto asociado con filogenia; y selección sexual. El $16 \%$ de respuestas desacertadas fue clasificado en: colonización; desarrollo y reproducción (la evolución como crecimiento); creacionismo; y confusión con conservacionismo (Cuadro 1).

Cuadro 1. Respuestas a las preguntas: ¿Cómo evolucionan los animales y las plantas? y ¿Qué es para ti la evolución?

\begin{tabular}{lcc}
\hline Respuestas acertadas & Total & Porcentaje \\
\hline Adaptación (a recursos y al clima) & 157 & 29 \\
Variación & 128 & 24 \\
Cambios a través del tiempo & 85 & 16 \\
Selección natural & 43 & 8 \\
Conceptos asociados con filogenia & 33 & 6 \\
Selección sexual & 5 & 1 \\
\hline Subtotal & $\mathbf{4 5 1}$ & $\mathbf{8 4}$ \\
\hline Respuestas desacertadas & & 6 \\
\hline Colonización & 31 & 6 \\
Desarrollo y reproducción & 31 & 3 \\
Creacionismo & 18 & 1 \\
Conservacionismo & 7 & $\mathbf{1 6}$ \\
\hline Subtotal & $\mathbf{8 7}$ & $\mathbf{1 0 0}$ \\
\hline Total & $\mathbf{5 3 8}$ & $\mathbf{6 0}$ \\
\hline
\end{tabular}

* Hubo una respuesta desacertada, clasificada como "Evolución como algo solo del pasado", que no se incluyó en el cuadro por su singularidad. 
Entre las respuestas acertadas destaca el amplio porcentaje relacionado con conceptos de "adaptación" y "variación", así como la identificación de la evolución como cambios a través del tiempo. En cuanto a la selección sexual, las siete respuestas en este sentido ilustran una influencia directa de la lectura de El misterioso, pues este tema no es abordado en el currículo educativo.

En relación con los desaciertos, tomamos nota de tres confusiones: la confusión con el concepto de colonización de islas, como si fuera parte directa de la evolución; la idea de que evolución es sinónimo de desarrollo del ser vivo; y la confusión con el conservacionismo.

En relación con los dibujos, se obtuvieron 225 , de los cuales se excluyeron once que no representaban ningún concepto (por ejemplo la silueta de un ser humano), y que resultaban inclasificables. Los restantes 214 dibujos fueron organizados en cuatro categorías: cambio en animales (35\%); cambio en humanos (12\%); dibujo copiado del libro (33\%); y dibujos desacertados (20\%); éstos últimos incluyeron los de organismos, procesos naturales, paisajes, incluso cometas, en los que no se apreciaba una interpretación de la evolución, así como los de colonización, desarrollo y reproducción, entre otros (Cuadro 2).

Cuadro 2. Respuestas a la instrucción: muestra con dibujos la teoría de la evolución de las especies.

\begin{tabular}{|c|c|}
\hline Categoría del dibujo & Total \\
\hline \multicolumn{2}{|l|}{ Cambio en animales } \\
\hline Pinzón & 19 \\
\hline Iguana (terrestre o marina) & 19 \\
\hline Ave & 13 \\
\hline Pingüino & 8 \\
\hline Iguana marina & 7 \\
\hline Cormorán & 4 \\
\hline Albatros & 2 \\
\hline Tortuga & 2 \\
\hline Fragata & 2 \\
\hline Animal acuático & 1 \\
\hline Lobo marino & 1 \\
\hline Cuadrúpedo & 1 \\
\hline Subtotal cambio animales & 76 \\
\hline Cambio en humanos & 26 \\
\hline Dibujo desacertado & 42 \\
\hline \multicolumn{2}{|l|}{ Dibujo copiado del libro } \\
\hline Estrategias colonización & 23 \\
\hline Especiación cucuves & 22 \\
\hline Iguana marina pensando otra estrategia & 16 \\
\hline Piqueros patas azules bailando & 3 \\
\hline Caricatura de Darwin & 3 \\
\hline Árbol de coral & 2 \\
\hline ¿Ave? & 1 \\
\hline Subtotal dibujo copiado & 70 \\
\hline TOTAL & 214 \\
\hline
\end{tabular}


Entre los dibujos inéditos de "Cambio en animales", destacan los de picos de pinzones y de iguanas; también que los nueve dibujos del endémico pingüino de Galápagos fueron hechos por estudiantes de la isla Isabela, donde los moradores del poblado, a diferencia de las otras islas, mantienen un contacto diario con estos animales. Esto indica la pertinencia de cierta fauna para la explicación de los procesos evolutivos en contexto; en este caso no son mamíferos ni criaturas terrestres, sino aves y animales nadadores a los que aludieron los estudiantes, y en el caso de los pingüinos, a los que están en su entorno inmediato.

En algunos casos la complejidad del dibujo fue sorprendente, como el de una interpretación de la evolución de la iguana marina a partir de un ancestro terrestre (Ilustración 2).

Preguntas 8, 9 y 10: ¿Qué te gustó más del libro?; ¿Qué te gustó menos?; Dibuja la parte del libro que te impresionó más

En relación con la pregunta abierta “¿qué te gustó más?”, los y las estudiantes dieron respuestas en ocho categorías: Ciencia (38\%); Aventura (36\%); Parte específica del libro (un pasaje en concreto, 10\%); Dibujos (7\%); y Formato literario (5\%). El restante 4\% se repartió entre: Todo, Inclasificable, y Charla al recibir el libro (Gráfico 3).

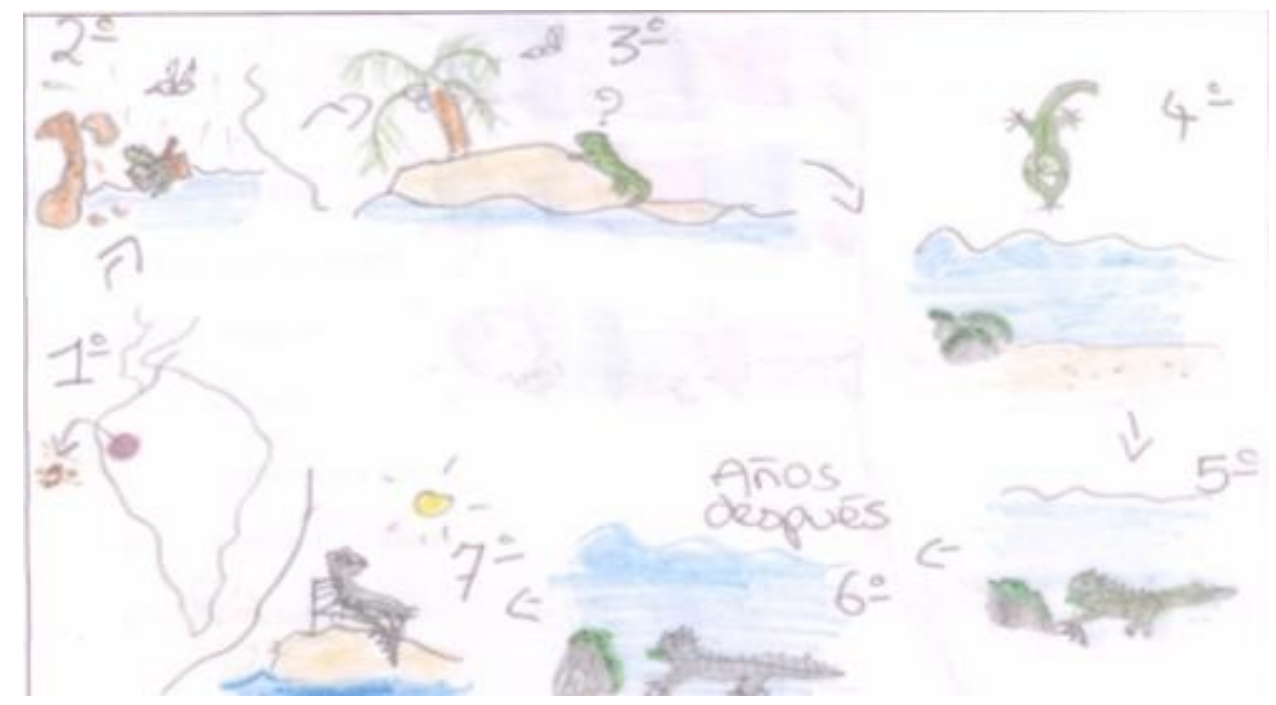

Ilustración 2. Dibujo de una estudiante sobre la evolución de la endémica iguana marina. Fuente: Encuesta 81, estudiante de $8^{\circ}$ grado, Escuela Tomás de Berlanga.
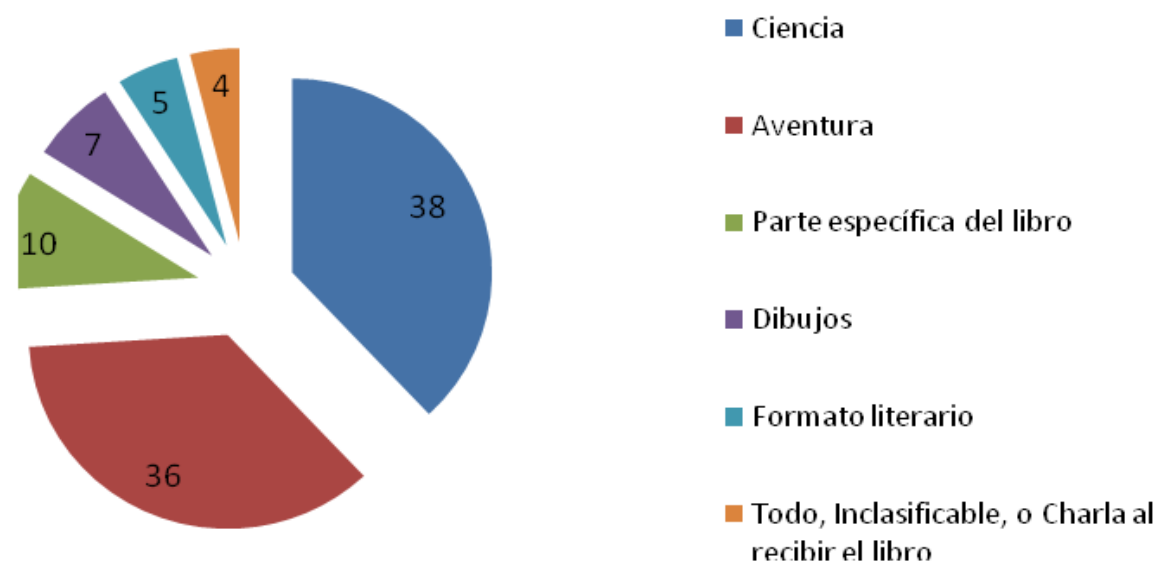

Gráfico 3. ¿Qué te gustó más del libro? 
Ante la pregunta "¿qué te gustó menos?" los estudiantes se refirieron a situaciones incómodas o peligrosas de la aventura, es decir, a pasajes específicos de la trama de ficción en las cuales la vida de los protagonistas corría peligro. Apenas siete respuestas aludieron a los conceptos científicos como algo negativo, lo cual, al compararse con el 38\% que afirma un gusto por la parte científica, insinúa que la ciencia en ficción es pertinente para acercar a las y los chicos galapagueños a las ciencias de la vida y, de modo general, al gusto por la actividad científica.

Preguntas 1, 2, 3 y 4 de la encuesta a profesores: ¿Le ha servido el libro para sus clases? ¿Cómo?; ¿Le parece una buena estrategia para enseñar sobre ecología y evolución?; ¿Qué le gustó más del libro? ¿Qué le gustó menos? ¿Qué cambiaria?; ¿Tiene algún comentario adicional?

Para ilustrar la perspectiva docente, destacamos en primer lugar una inesperada respuesta proveniente de la Escuela Adventista:

"Aunque de manera muy personal creo en la Creación, considero que es importante conocer la teoría de la evolución y demás teorias porque nuestra responsabilidad como maestros es guiar de la mejor manera posible a los estudiantes y proporcionarles todas las herramientas para que desarrollen un pensamiento crítico y autónomo” (Encuesta 1).

La mayoría de profesores coincidió en la utilidad del libro para sus clases de literatura y de ciencias, y sobre su calidad literaria, destacando la pertinencia de que la historia ocurra en un escenario familiar, que los protagonistas sean jóvenes, que uno de ellos siga estudiando y se supere, entre otros. Algunos comentarios en esta línea fueron:

\footnotetext{
"El libro introduce a la teoría de la evolución con un vocabulario sencillo pero a la vez científico permitiendo a niños y niñas conocer sobre este tópico con facilidad, por lo que si me ha servido en mis clases de ciencias naturales [...] Es una excelente estrategia porque conjuga aspectos de la vida cotidiana galapagueña con situaciones fantásticas que permiten al estudiante involucrarse en la lectura con gran facilidad" (Encuesta 6).

'Lo que más me gustó fue la combinación perfecta que hay entre narrativa, ecología y evolución, y sobre todo que el final no es predecible" (Encuesta 7).

"[...] las acciones y escenarios son muy familiares y ello permite activar eficientemente los contenidos a tratar, y sobre todo porque son personajes en edades similares a este grupo" (Encuesta 5).
}

También comentaron sobre su utilidad para fomentar una cultura conservacionista de la naturaleza, por ejemplo:

"Es un excelente libro que nos ayuda a conocer sobre Darwin y especialmente a los jóvenes les llamó mucho la atención y ellos mismos pedian leer. Lo bicimos en el aula y comentamos y reflexionamos sobre el valor de cuidar nuestras islas" (Encuesta 4).

Un reclamo frecuente de los docentes fue el de más ilustraciones. También hubo críticas al exceso de fantasía. Pero para la mayoría fue adecuado, al igual que lo fue para los estudiantes.

\section{Conclusiones}

Las conclusiones a continuación están organizadas en torno a las cuatro preguntas planteadas al inicio, relacionadas con nuestra curiosidad por conocer el proceso de recepción de El misterioso reloj de Darwin entre algunos estudiantes y profesores de Galápagos: ¿Qué conocimiento tienen los estudiantes galapagueños sobre quién fue Charles Darwin y sobre aspectos relacionados con la teoría de la evolución? ¿Es la ciencia en ficción un recurso eficiente para apoyar y fortalecer a la educación formal en torno a la literatura, la historia y las ciencias de la vida en el contexto galapagueño? ¿Qué aprendizajes quedan de esta experiencia educativa? ¿Cómo se articula esta experiencia con los debates en torno a la construcción de una Tercera Cultura? 
Este análisis, conviene recordarlo, está basado en los resultados de una encuesta exploratoria, que arroja indicios exclusivamente sobre la muestra consultada, y cuyos resultados no pueden ser extrapolados al conjunto de la población escolar de las islas.

En primer lugar, se constató que los estudiantes galapagueños encuestados tienen muy buenos conocimientos sobre quién fue Charles Darwin y qué hizo en Galápagos. Del mismo modo, la mayoría identifica las estrategias que tuvo la fauna para colonizar las islas, una noción clave para entender los procesos evolutivos.

En relación con la comprensión de la teoría de la evolución (o aspectos clave de ésta), muchos tienen nociones acertadas, aunque la presencia de una gran cantidad de respuestas en blanco y de otras equivocadas (que suman casi un tercio de la muestra), apunta a la necesidad de fortalecer la educación sobre este tema en todos los niveles.

En segundo lugar, los resultados sugieren que la ciencia en ficción (pese a muy puntuales resistencias) es pertinente para enseñar historia y evolución en contextos de educación formal. Entre los encuestados, El misterioso reloj de Darwin, fortaleció los conocimientos sobre quién fue Darwin y sobre aspectos de la teoría de la evolución, creando una buena disposición para el contacto con las explicaciones formales del origen de las especies. Desde el punto de vista metodológico, la estrategia de usar ciencia en ficción parece ser una novedosa motivación para estudiantes y profesores, para aprender sobre su territorio, sobre historia de la ciencia, sobre ciencia y sobre las características de la actividad científica.

Un aprendizaje importante de esta experiencia es ratificar el valor que tiene la enseñanza de la ciencia en contexto. No basta con llevar a cualquier lugar, como Galápagos, productos de divulgación científica elaborados en territorios diferentes, aunque se trate de productos con contenidos universales, como la teoría de la evolución; es necesario que los contenidos estén acoplados a la realidad inmediata. Ahora bien, un libro de ciencia en ficción no puede hacerlo todo para que los y las adolescentes aprendan sobre evolución; el éxito también depende de la articulación que la docencia haga con otras actividades y de su preparación para lidiar con este tema. Pero si hay lugares "ideales" en los cuales es posible echar mano de ejemplos in situ para aprender sobre evolución, uno de éstos es Galápagos. Allí la evolución, y de modo general las ciencias de la vida, pueden adquirir una relevancia amplia, transversal, en los procesos de aprendizaje.

Futuras intervenciones y estudios relacionados con el aprendizaje de la evolución en Galápagos (y otros contextos) podrían apuntar a indagar de forma más precisa el alcance del entendimiento de la teoría en torno a conceptos específicos como "tiempo geológico", "selección natural", "adaptación", "evolución” o "genética". También parece indispensable indagar las articulaciones construidas con el pensamiento creacionista.

Para evaluaciones futuras recomendamos realizar encuestas antes y después de la entrega del material, algo que no estuvo a nuestro alcance. Más allá de las dificultades metodológicas, sin embargo, resulta relevante sistematizar y difundir los resultados de experiencias educativas impulsadas desde una institución productora de conocimiento científico, como la Fundación Charles Darwin en Galápagos, para fortalecer los procesos educativos locales.

Finalmente, como El misterioso fue usado en clases de ciencias y de literatura indistintamente, y tuvo éxito en todas, concluimos que la ciencia en ficción es una buena estrategia para mestizar ciencia y humanidades, rompiendo las tradicionales fronteras disciplinarias y fomentando la construcción de una Tercera Cultura. Incluso se organizó, en la isla Isabela, después de que realizamos esta evaluación, un concurso de pintura sobre la obra. El libro abrió a los estudiantes la posibilidad de apreciar la complementariedad en el conocimiento, refrescando el (a veces) impermeable currículo educativo. 


\section{Agradecimientos}

Agradecemos a Paola Espinosa por la tabulación de los datos y por sus aportes analíticos; y a Paz Guarderas por sus agudas observaciones. También al editor y a los lectores pares de la revista Eureka, cuyas sugerencias enriquecieron el contenido y la presentación del artículo. La producción de El misterioso reloj de Darwin y la realización de las encuestas fueron posibles gracias a la Fundación Charles Darwin; el análisis se hizo con el apoyo del Programa de Estudios Socioambientales de FLACSO-Ecuador. Esta publicación corresponde con la contribución número 2062 de la Fundación Charles Darwin para las Islas Galápagos.

\section{Referencias bibliográficas}

Alters, B. J. y Nelson, C. E. (2002). Perspective: Teaching Evolution in Higher Education. Evolution, 10(56), 1891-1901.

Bizzo, N. (2008). A percepção das crianças sobre fenômenos evolutivos: o que pensam jovens que se deparam com fósseis todos os dias? En L. Massarani (Ed.). Ciência \& criança: a divulgaşão científica para o público infanto-juvenil. Río de Janeiro: Museu da Vida, Casa de Oswaldo Cruz y Fiocruz.

Blanco López, Á. (2004). Relaciones entre la educación científica y la divulgación de la ciencia. Revista Eureka sobre Enseñanza y Divulgación de las Ciencias, 2(1), 70-86.

Caldwell, R., Collins, J., Frankel, J., et al. (Sin fecha). Comprendiendo la evolución para profesores. University of California Museum of Paleontology y National Center for Science Education. En línea en: http://www.sesbe.org/evosite/evohome.html, visita 15 agosto 2010.

Capra, F. (2002 [1996]). La trama de la vida. Una nueva perspectiva de los sistemas vivos. Barcelona: Anagrama.

Cuvi, N. (2010). El misterioso reloj de Darwin. Quito: Fundación Charles Darwin.

Darwin, C. R. (1987 [1876-1882]). Autobiografía. Recuerdos del desarrollo de mi mente y carácter. Barcelona: Alta Fulla.

Djerassi, C. (1996 [1994]). El gambito de Bourbaki. México: Fondo de Cultura Económica.

Djerassi, C. (2003 [1998]). NO. México: Fondo de Cultura Económica.

Dobzhansky, T. (1973). Nothing in Biology Makes Sense except in the Light of Evolution. The American Biology Teacher, 3(35), 125-129.

Ecsite (European network of science centres and museums). (2012). KïCS. En http://www.ecsite.eu/activities_and_resources/projects/kiics, visita 30 marzo 2012.

Erren, T. C. y Falaturi, P. (2009). Research insights and insides:"Science-in-Fiction”as a contribution to the Third Culture Concepts. Medical Hypotheses, 5(72), 487-490.

Fundación Charles Darwin (2008). Día Darwin, 2008. Puerto Ayora: Fundación Charles Darwin. Informe inédito.

Grenier, C. (2007). Conservación contra natura. Las islas Galápagos. Quito: Instituto Francés de Estudios Andinos, Embajada de Francia en el Ecuador, Institut de Recherche Pour le Devélopment, Universidad Andina Simón Bolívar y Abya-Yala.

INEC (Instituto Nacional de Estadísticas y Censos) (2012). Resultados del Censo de Población. En http://www.inec.gov.ec/cpv/, visita 27 de enero de 2012. 
Jackson, M. H. (2007 [1997]). Galápagos. Una historia natural. Sin ciudad: University of Calgary Press.

Jay Gould, S. (2004 [2003]). Érase una vez el zorro y el erizo. Las bumanidades y la ciencia en el tercer milenio. Barcelona: Drakontos.

Jensen, M. S. y Finley, F. N. (1995). Teaching evolution using historical arguments in a conceptual change strategy. Science Education, 2(79), 147-166.

Massarani, L., de Castro Moreira, I. y Almeida, C. (2006). Para que um diálogo entre ciência e arte? História, Ciências, Saúde - Manguinhos, Suplemento(13), 7-10.

Mateos Maroto, F. J. (2004). La evolución,... a escena. De cómo el grupo Prometeo enseña aspectos sobre la evolución y de los recursos que pueden emplearse para ello. Revista Eureka sobre Enseñanza y Divulgación de las Ciencias, 2(1), 122-135. En línea en: http://reuredc.uca.es.

Ministerio de Educación del Ecuador (2011a). Área de Ciencias Naturales. Actualización y Fortalecimiento Curricular de la Educación General Básica 2010. 8. ${ }^{\circ}$ 9. 9. y 10. ${ }^{\circ}$ años. Quito, Ministerio de Educación. En http://www.educacion.gob.ec/index.php/ actualizacion-curricular, visita 29 de enero de 2012.

Ministerio de Educación del Ecuador (2011b). Ciencias Naturales 9. De acuerdo al nuevo currículo de la Educación General Básica. Texto para estudiantes. Quito: Norma. En http://www.educacion.gob.ec/index.php/textos-escolares, visita 28 de enero de 2012.

Ministerio de Educación del Ecuador (2011c). Estadística Estudiantil de inicio de año lectivo 2011-2012. Puerto Baquerizo Moreno: Dirección Provincial de Educación de Galápagos, Coordinación de Estadística. 1 hoja.

Nieto-Galan, A. (2011). Los públicos de la ciencia. Expertos y profanos a través de la historia. Madrid: Fundación Jorge Juan y Marcial Pons.

Osborne, P. (1998). Migration - Utopia or myopia? Rangifer, 3-4(18), 155-156.

Palacios, S. L. (2007). El cine y la literatura de ciencia ficción como herramientas didácticas en la enseñanza de la física: una experiencia en el aula. Revista Eureka sobre Enseñanza y Divulgación de las Ciencias, 1(4), 106-122.

Sierra Cuartas, C. E. (2008). Bioética y ensayística: nexos y cautelas para la enseñanza e investigación de la Bioética. Revista Latinoamericana de Bioética, 2(8), 88-105.

Tidon, R. y Lewontin, R. C. (2004). Teaching evolutionary biology. Genetics and Molecular Biology, 1(27), 124-131.

Weiner, J. (1994). The beak of the finch: a story of evolution in our time. Nueva York: Knopf.

Wilson, E. O. (1999 [1998]). Consilience: la unidad del conocimiento. Barcelona: Galaxia Gutenberg y Círculo de Lectores.

Winter, E. (2004). Public Communication of Science and Technology: German and European Perspectives. Science Communication, 3(25), 288-293. 


\section{Anexo 1: encuesta aplicada a estudiantes}

Nombre de la institución; Grado; Fecha.

1) ¿Cuántos libros has leído?

Ninguno; 1 libro; entre 2 y 3 libros; entre 4 y 5 libros; más de 5 libros.

2) ¿Leíste El misterioso reloj de Darwin?

No lo leí; Sólo el inicio; Un poco menos de la mitad; Un poco más de la mitad; Todo el libro.

3) ¿Quién fue Charles Darwin y qué hizo en su visita a Galápagos?

Un turista que vino a pasear; Un joven cientifico que vino a investigar la flora y la fauna; Un marinero que llegó sin querer a Galápagos; El capitán del Beagle que vino a dibujar mapas de Galápagos; Un científico de barba que vino varias veces a Galápagos.

4) ¿Cómo llegaron los ancestros de las plantas y animales propios de Galápagos? (escoge 2 respuestas)

En los barcos de los piratas; En balsas de vegetación y arrastradas por los vientos; La gente los trajo; Siempre vivieron aqui; Volando, nadando y flotando.

5) De acuerdo con la información del libro, ¿cómo evolucionan los animales y plantas?

6) Después de leer El misterioso reloj de Darwin, ¿qué es para ti la evolución?

7) Muestra con dibujos la teoría de la evolución de las especies.

8) ¿Qué te gustó más del libro?

9) ¿Qué te gustó menos?

10) Dibuja la parte del libro que te impresionó más.

\section{Anexo 2: encuesta aplicadas a profesores}

Nombre de la institución; Grado; Fecha.

1. ¿Le ha servido el libro para sus clases? ¿Cómo?

2. ¿Le parece una buena estrategia para enseñar sobre ecología y evolución?

3. ¿Qué le gustó más del libro? ¿Qué le gustó menos? ¿Qué cambiaría?

4. ¿'Tiene algún comentario adicional? 